\title{
Mobility Choices and Strategic Interactions in a Two-Group Macroeconomic-Epidemiological Model
}

\author{
Davide La Torre $^{1}$ (D) Danilo Liuzzi ${ }^{2} \cdot$ Rosario Maggistro $^{3} \cdot$ Simone Marsiglio $^{4}$
}

Accepted: 1 November 2021 / Published online: 2 December 2021

(c) The Author(s), under exclusive licence to Springer Science+Business Media, LLC, part of Springer Nature 2021

\begin{abstract}
We analyze the implications of strategic interactions between two heterogeneous groups (i.e., young and old, men and women) in a macroeconomic-epidemiological framework. The interactions between groups determine the overall prevalence of a communicable disease, which in turn affects the level of economic activity. Individuals may lower their disease exposure by reducing their mobility, but since changing mobility patterns is costly, each group has an incentive to free ride negatively affecting the chances of disease containment at the aggregate level. By focusing on an early epidemic setting, we explicitly characterize the cooperative and noncooperative equilibria, determining how the inefficiency induced by noncooperation (i.e., failure to internalize epidemic externalities) depends both on economic and epidemiological parameters. We show that long-run eradication may be possible even in the absence of coordination, but coordination leads to a faster reduction in the number of infectives in finite time. Moreover, the inefficiency induced by noncooperation increases (decreases) with the factors increasing (decreasing) the pace of the disease spread.
\end{abstract}

Keywords Dynamic programming - Infectious diseases · Macroeconomic outcomes · Mobility choices $\cdot$ Noncooperative versus cooperative games

Mathematics Subject Classification 91A10 · 91A12 · 91A25 · 91B64

JEL Classification C60 $\cdot$ C70 $\cdot$ E20 $\cdot$ I10

This article is part of the topical collection "Modeling and Control of Epidemics" edited by Quanyan Zhu, Elena Gubar and Eitan Altman.

We are grateful to two anonymous referees for their constructive comments on earlier draft of the paper. We also wish to thank Nahid Masoudi for insightful discussions. All remaining errors and omissions are our own sole responsibility. Simone Marsiglio's research has been supported by the Italian Ministry of University and Research as a part of the PRIN 2017 program (grant protocol number 2017FKHBA8), and by the University of Pisa under the "PRA - Progetti di Ricerca di Ateneo" (Institutional Research Grants) - Project no.

PRA_2020_79 "Sustainable development: economic, environmental and social issues".

Data sharing not applicable to this article as no datasets were generated or analyzed during the current study.

Extended author information available on the last page of the article 


\section{Introduction}

Infectious diseases represent still today a major source of morbidity and mortality in both developing and industrialized countries, crucially affecting their prospects for economic development $[28,34]$. Through their effects on health conditions and thus the pressure on governments to finance health policy, the impact on communicable diseases extends to macroeconomic outcomes affecting the labor market, productivity, saving and investment decisions $[8,26]$. The analysis of such a health-economic relation is the focus on the economic epidemiology literature which seeks to understand the effectiveness of different policy tools in mitigating the economic consequences of infectious diseases [17,31], along with their implications on macroeconomic dynamics and economic prosperity $[18,26]$. Despite their differences in the economic framework, these papers share the same epidemiological setting, given by the susceptible-infected-susceptible (SIS) model, in which individuals are either susceptible to the disease or already infected and infectives, without ever acquiring permanent immunity from infection [21,24]. The SIS framework represents one of simplest models in mathematical epidemiology and is widely applicable to a range of traditional diseases, such as the seasonal flu and the common cold, but also to novel diseases such as COVID-19, since thus far there is no convincing evidence that after recovery individuals may become immune from a second infection [35].

The ongoing COVID-19 pandemic is ravaging the entire planet showing more clearly than ever that sudden and unforeseeable epidemic episodes can dramatically hit not only developing countries but even the industrialized ones. The most widely used mitigation strategies (i.e., social distancing and lockdowns) have not only generated beneficial effects on disease incidence and prevalence, but also devastating economic consequences by forcing individuals and firms to limit their social contacts and interactions both on the workplace and at home. These striking facts have spurred a growing interest in understanding the mutual relation between epidemic and macroeconomic outcomes, and several works have explored the macroeconomic implications of COVID-19 and the policy measures implemented to control it $[1,3,5,25]$. Most of the papers analyze policymakers' optimal response by determining from a normative point of view the public policy tools to employ in order to achieve the first best outcome, while limited has been thus far the focus on individuals' voluntary choices to reduce their disease exposure. However, several works document that individuals have significantly reduced their mobility in an attempt to decrease their contacts with potentially infective individuals $[9,19]$. To the best of our knowledge, very few papers have tried thus far to characterize individuals' response to the level of disease prevalence, discussing how their behavioral change may improve health outcomes at the cost of deteriorating the economic ones [16]. In this paper, we wish to contribute to this literature by analyzing the behavioral response to disease prevalence of different demographic groups and what the interaction between groups may imply for epidemic and macroeconomic outcomes.

One of most evident and potentially long-lasting economic consequences of COVID-19 concerns its heterogeneous impact on different population groups. The widespread adoption of lockdown and social distancing measures, including school closures and remote working arrangements, has generated important labor market shocks leading to a substantial income reduction for a large share of the population resulting in growing concerns for the future and a deterioration in mental health conditions $[11,27]$. The labor market and income consequences have been highly heterogeneous, hitting particularly younger workers, those with precarious employment and from minority ethnic groups, along with individuals employed in contactintensive sectors precluded from remote working [4,15]. Moreover, such economic impacts 
have also been highly heterogeneous across gender and age: Women have suffered more than men due to their traditional role as primary carer for children [2,22], while young people have suffered more than old individuals as they have experienced more radical changes to their lives and have gained less from disease containment measures [6,20]. Apart from the direct and indirect implications of public policy, also the mitigation strategies voluntarily employed within the population in order to reduce the risk of disease exposure have been highly heterogeneous, and in particular women and the young have reduced their mobility by larger amounts than men and the old, suggesting that overall gender and inter-generational inequalities have been and may be widening during the COVID-19 crisis [7]. Understanding thus how different demographic groups (i.e., men and women, young and old) may modify their behavior following an epidemic shock is essential in order to shed some light on the possible consequences of the COVID-19 pandemic on social inequalities and to develop effective policy strategies.

In order to move forward in this direction, we develop a macroeconomic-epidemiological model in which two heterogeneous population groups decide how to voluntarily modify their behavior to lower their risk to contract the disease. Specifically, individuals in each group choose the extent to which reducing their mobility, but changing mobility patterns is costly since by affecting both production and leisure it decreases consumption and the enjoyment of recreational services. Each group needs to balance the competing health (i.e., decrease new infections) and economic (i.e., increase consumption and enjoy recreation) needs, moved by an incentive to free ride in order to let the costly behavioral change fall on the other group, which may result in a deterioration of the chances to contain the disease spread at the aggregate level. We focus on an early epidemic setting in which the number of infectives follows an exponential dynamics and both groups' susceptible shares are proxied by their relative population shares. This gives rise to a two-player differential game in which players differ in some key epidemiological and economic factors and strategically interact in the pursuit of their own self-interest. Differential games have been extensively employed to analyze a variety of issues in environmental and natural resources management, industrial organization, epidemiology $[23,30,32]$, but to the best of our knowledge applications in economic epidemiology have not been discussed yet. Specifically, in our macroeconomic-epidemiological differential game we analyze the players' mobility choices and disease dynamics both under cooperation and noncooperation, comparing the equilibrium outcomes under such different scenarios. We characterize how the noncooperative and cooperative equilibrium outcomes depend on both economic and epidemiological parameters, determining how such parameters impact the inefficiency induced by noncooperation (i.e., the failure to internalize the epidemic externalities that the two groups impose on each other). We show that in all scenarios the burden of reducing mobility patterns falls more strongly on the group characterized by a smaller population share and lower adjusted productivity (i.e., lower share of time allocated to labor activities), and cooperation helps redistributing this burden between groups. We also show that long-run eradication may be possible even in the absence of coordination, but coordination leads to a faster reduction in the number of infectives in finite time, and that the distortion induced by noncooperation increases with the factors increasing the pace of the disease spread (i.e., the infectivity rate) and decreases with those reducing it (the recovery rate and the adjusted productivity parameter).

Our paper makes thus a number of novel contributions to the literature. To the best of our knowledge, it is the first work analyzing strategic interactions between groups in a differential game setting in the context of macroeconomic epidemiology. Moreover, it develops a tractable framework which, given the specific linear-quadratic structure of the problem under an early epidemic stage assumption, allows for closed-form solutions explicitly determining 
the players' optimal closed-loop strategies and the optimal evolution of disease prevalence. This permits us to derive interesting (and to some extent surprising) conclusions: (i) Eradication may be possible even in the absence of coordination, meaning that despite their free-riding incentives the strategic interactions between groups may not preclude the achievement of the long-run eradication goal; (ii) the burden of implementing behavioral changes to limit the spread of the disease affects different groups unequally, and specifically, the impact of such a burden depends on key groups' demographic and economic characteristics. Such results have important policy implications which can inform policymakers' efforts to mitigate the heterogeneous economic consequences of infectious diseases between different population groups.

The paper proceeds as follows. Section 2 introduces our macroeconomic-epidemiological differential game in which individuals of two groups decide the extent to which reducing their mobility in order to lower their infection risk and such a choice affects also their consumption and recreation. Section 3 characterizes the cooperative and noncooperative equilibrium outcomes, deriving explicitly the players' mobility reduction choices in both frameworks and relying on numerical experiments to compare the cooperative and noncooperative solutions. Section 4 analyzes the special case in which heterogeneity between the groups is ruled out in order to derive explicitly some results which would otherwise be obtained only through numerical analysis. Section 5 as usual presents concluding remarks and highlights directions for future research. All mathematical technicalities are presented in Appendix A.

\section{The Model}

We consider a macroeconomic-epidemiological differential game in which two groups (i.e., young and old, men and women) by interacting with each other determine the overall prevalence of a communicable disease and thus the level of economic activity. Individuals may lower their risk to contract the disease by reducing their mobility, but since changing mobility patterns is costly, each group has an incentive to free ride negatively affecting the chances of disease containment at the aggregate level. A similar setting has been recently analyzed in [25] in a single group context to determine the optimal social distancing policy, abstracting thus from a game structure and the implications of strategic interactions on disease dynamics and macroeconomic outcomes.

\subsection{The Epidemiological Framework}

We consider a two-group SIS framework in which the individuals of each group can be either infectives or susceptible to the disease, but they cannot acquire permanent immunity. Abstracting from vital dynamics, the total population $N$ is assumed to be constant and to be composed by individuals of group $i$ and group $j$ only: $N=N^{i}+N^{j}$. In the following, we shall present the model's structure and equations only for group $i$, but the same structure and equations apply symmetrically to group $j$. Individuals in group $i, N^{i}$, can be either healthy but susceptible to infection $S_{t}^{i}$ or infectives who can transmit the disease by getting in contact with susceptibles (of both groups) $I_{t}^{i}: N^{i}=S^{i}+I^{i}$. The interactions between susceptibles of a group and infectives of both groups determine the evolution of susceptibles and infectives for a given group. For the sake of simplicity, independently of the group they belong to, infectives spontaneously recover at the rate $\delta>0$, and susceptibles become infective by interacting with infectives at the rate $\alpha>0$, measuring the number of social 
contacts required to give rise to a new infection. Therefore, for group $i$, given its initial levels $S_{0}^{i}, I_{0}^{i} \geq 0$, the dynamics of susceptibles and infectives is given by the following equations:

$$
\begin{aligned}
& \dot{S}_{t}^{i}=\delta I_{t}^{i}-\alpha S_{t}^{i}\left(\frac{I_{t}^{i}+I_{t}^{j}}{N}\right) \\
& \dot{I}_{t}^{i}=\alpha S_{t}^{i}\left(\frac{I_{t}^{i}+I_{t}^{j}}{N}\right)-\delta I_{t}^{i},
\end{aligned}
$$

with $j \neq i$. Note that in the above equations the total number of infectives $I_{t}^{i}+I_{t}^{j}$ which can generate new infections is normalized by the total population size $N$ to represent that the patterns of social interactions between individuals tend to remain stable over time and do not change with the level of disease prevalence [18]. Since $N^{i}=S^{i}+I^{i}$, it follows that one of the two equations above is redundant and thus it is possible to focus only on one of them. By focusing on infectives, from $S^{i}=N^{i}-I^{i}$ we can simplify the above system as follows:

$$
\dot{I}_{t}^{i}=\alpha\left(N^{i}-I^{i}\right)\left(\frac{I_{t}^{i}+I_{t}^{j}}{N}\right)-\delta I_{t}^{i} .
$$

By defining the share of infectives, susceptibles and population out of the total population for each group as $i^{i}=\frac{I^{i}}{N}, s^{i}=\frac{S^{i}}{N}$ and $n^{i}=\frac{N^{i}}{N}$, respectively, we can recast the evolution of infectives in group $i$ as follows:

$$
\dot{i}_{t}^{i}=\alpha\left(n^{i}-i_{t}^{i}\right)\left(i_{t}^{i}+i_{t}^{j}\right)-\delta i_{t}^{i} .
$$

The expression above, which applies symmetrically also to group $j$, suggests that the evolution of infectives in both groups depends on the same parameters and the total number of infectives between groups determines the disease incidence in each group. From a mere epidemiological perspective, the two groups are homogeneous apart from their relative population shares; that is, $n^{i}$ and $n^{j}=1-n^{i}$ are the only source of heterogeneity between groups as all other parameters ( $\alpha$ and $\delta$ ) are assumed to be the same for both groups.

\subsection{The Macroeconomic Framework}

We now extend the above epidemiological framework to account for how individuals' decisions to lower their infection exposure may impact disease dynamics and macroeconomic outcomes. Specifically, in order to reduce the number of persons they get in contact with, individuals may decrease their mobility (i.e., working from home rather than on the workplace and meeting friends virtually rather than in person) by a certain share $0<u^{i}<1$ lowering thus the degree of infectivity and disease incidence, but also their productivity both in the production and leisure sectors (i.e., most production processes and leisure activities require face-to-face interactions and although online interactions are possible, they often are less effective than the physical ones). Individuals exogenously split their unitary time endowment between working in the production sector and enjoying leisure, resulting in the production of goods and recreational services, respectively. Firms produce competitively the unique consumption good by employing labor, while recreational services are produced as a side-effect of leisure activities. Only susceptibles work and enjoy leisure, while infectives are quarantined and receive income support financed via income taxes levied on susceptibles. Households entirely consume their disposable income as follows: $c_{t}^{i}=q_{t}^{i}-\tau$, where $c_{t}$ denotes consumption, $q_{t}$ income and $\tau>0$ a lump-sum tax used to provide income support 
to infectives. The consumption good is produced through a linear production technology depending only on the amount of time allocated to labor, and individuals' reduction mobility choices negatively affect productivity as follows: $q_{t}^{i}=\beta \phi^{i}\left(1-u_{t}^{i}\right) s_{t}^{i}$, where $\beta>0$ measures the productivity and $0<\phi^{i}<1$ is the share of time allocated to labor activities. The share of nonworking time $0<1-\phi^{i}<1$ is allocated to leisure through which recreational services are produced (and instantaneously enjoyed), and also in this case, individuals' reduction mobility choices negatively affect productivity as follows: $r_{t}^{i}=\theta\left(1-\phi^{i}\right)\left(1-u_{t}^{i}\right) s_{t}^{i}$, where $r_{t}^{i}$ is the recreational service output and $\theta>0$ is the productivity parameter. Individuals' change in mobility patterns generates thus a personal loss $x_{t}^{i}$ equal to the consumption and recreational losses given by: $x_{t}^{i}=u_{t}^{i} q_{t}^{i}+u_{t}^{i} r_{t}^{i}$. The disease dynamics is described by the following SIS equation: $i_{t}^{i}=\alpha\left(1-u_{t}^{i}\right) s_{t}^{i}\left(i_{t}^{i}+i_{t}^{j}\right)-\delta i_{t}^{i}$ where individuals' reduction mobility choices by lowering potentially infectious contacts slow down the disease transmission. Individuals seek to minimize the personal cost from their behavioral change, which is given by the discounted sum ( $\rho>0$ is the rate of time preference) of the instantaneous losses. The instantaneous loss function is assumed to depend on the total spread of the disease $i_{t}^{i}+i_{t}^{j}$ and the personal loss due to the reduced mobility $x_{t}=\left[\beta \phi^{i}+\theta\left(1-\phi^{i}\right)\right] u_{t}^{i} s_{t}^{i}$ and to take a quadratic nonseparable form as follows [25,26]: $\ell^{i}\left(i_{t}^{i}+i_{t}^{j}, x_{t}^{i}\right)=\frac{\left(i_{t}^{i}+i_{t}^{j}\right)^{2}\left[1+\left(x_{t}^{i}\right)^{2}\right]}{2}$, penalizing deviations from the disease-free status (i.e., $i_{t}^{i}+i_{t}^{j}=0$ ) and from the no-personal-loss scenario (i.e., $x_{t}^{i}=0$ ).

By recalling that $s_{t}^{i}=n^{i}-i_{t}^{i}$, the decision problem of group $i$ can be summarized as follows:

$$
\begin{aligned}
\min _{u_{t}^{i}} & \mathcal{C}^{i}=\int_{0}^{\infty} \frac{\left(i_{t}^{i}+i_{t}^{j}\right)^{2}\left[1+\left(\xi^{i}\right)^{2}\left(u_{t}^{i}\right)^{2}\left(n^{i}-i_{t}^{i}\right)^{2}\right]}{2} e^{-\rho t} d t \\
\text { s.t. } & i_{t}^{i}=\alpha\left(1-u_{t}^{i}\right)\left(n^{i}-i_{t}^{i}\right)\left(i_{t}^{i}+i_{t}^{j}\right)-\delta i_{t}^{i} \\
& i_{t}^{j}=\alpha\left(1-u_{t}^{j}\right)\left(n^{j}-i_{t}^{j}\right)\left(i_{t}^{i}+i_{t}^{j}\right)-\delta i_{t}^{j} \\
& 0 \leq i_{t}^{i}, i_{t}^{j} \leq 1 \\
& i_{0}^{i}>0, i_{0}^{j}>0 \text { given }
\end{aligned}
$$

where $\xi^{i}=\beta \phi^{i}+\theta\left(1-\phi^{i}\right)$ measures the total time-allocation-adjusted productivity of group $i$ in the production and leisure sectors, which in the following we shall refer to as "adjusted productivity" for the sake of expositional simplicity. From an economic perspective, the two groups differ only by the parameters $\xi^{i}$ and $\xi^{j}$ via their different time allocation between sectors: As the productivity parameters $\beta$ and $\theta$ are assumed to be homogeneous between groups, the only source of heterogeneity is represented by the groups' time allocation. For the sake of simplicity, we assume that for each group the productivity is higher in the production than in the leisure sector (i.e., $\beta>\theta$ ) such that its adjusted productivity and sectoral time allocation $\xi^{i}$ and $\phi^{i}$ (and the same is true for $\xi^{j}$ and $\phi^{j}$ ) are positively related.

\subsection{The Early Epidemic Framework}

Since policymakers take time to understand how to respond to a new epidemic outbreak, every epidemic dynamic is characterized by an early phase in which the disease spreads freely across the population and individuals' behavioral reactions to the disease prevalence represent the main form of response to the epidemic. In our analysis, we specifically focus on this setting which seems to best fit our model's assumptions in which the effects of public 
policy (in the form of prevention or treatment interventions) are completely ruled out. In an early epidemic phase, the number of infectives tends to grow at a constant rate and thus the disease dynamics can be described by a linear differential equation by proxying both groups' susceptible shares by their relative population shares, $s_{t}^{i}=n^{i}-i_{t}^{i} \simeq n^{i}$ and $s_{t}^{j}=n^{j}-i_{t}^{j} \simeq n^{j}$ $[10,25,29]$. Under such an assumption, the group $i$ 's problem boils down to the following:

$$
\begin{aligned}
\min _{u_{t}^{i}} & \mathcal{C}^{i}=\int_{0}^{\infty} \frac{\left(i_{t}^{i}+i_{t}^{j}\right)^{2}\left[1+\left(\xi^{i}\right)^{2}\left(u_{t}^{i}\right)^{2}\left(n^{i}\right)^{2}\right]}{2} e^{-\rho t} d t \\
\text { s.t. } & i_{t}^{i}=\alpha n^{i}\left(1-u_{t}^{i}\right)\left(i_{t}^{i}+i_{t}^{j}\right)-\delta i_{t}^{i} \\
& \dot{i}_{t}^{j}=\alpha n^{j}\left(1-u_{t}^{j}\right)\left(i_{t}^{i}+i_{t}^{j}\right)-\delta i_{t}^{j} \\
& 0 \leq i_{t}^{i}, i_{t}^{j} \leq 1 \\
& i_{0}^{i}>0, i_{0}^{j}>0 \text { given }
\end{aligned}
$$

The optimization problem above states that the total disease prevalence drives the personal cost associated with individual mobility choices and the disease incidence for each group which ultimately determines the group-specific level of disease prevalence. Since for each group, both in the objective function and in the dynamic constraints, what really matters is the total disease prevalence, by defining $i_{t}=i_{t}^{i}+i_{t}^{j}$ and by recalling $n^{i}+n^{j}=1$, we can recast group $i$ 's problem as follows:

$$
\begin{aligned}
\min _{u_{t}^{i}} & \mathcal{C}^{i}=\int_{0}^{\infty} \frac{i_{t}^{2}\left[1+\left(\xi^{i}\right)^{2}\left(u_{t}^{i}\right)^{2}\left(n^{i}\right)^{2}\right]}{2} e^{-\rho t} d t \\
\text { s.t. } & \dot{i}_{t}=\left[\alpha\left(1-u_{t}^{i}\right) n^{i}+\alpha\left(1-u_{t}^{j}\right) n^{j}-\delta\right] i_{t} \\
& 0 \leq i_{t} \leq 1 \\
& i_{0}>0 \text { given }
\end{aligned}
$$

Note that such an early epidemic setting assumption, jointly with our specification of the instantaneous loss function, provides our model with a linear-quadratic structure which allows for closed-form solutions explicitly determining the closed-loop strategies of the two groups and the evolution of disease prevalence. ${ }^{1}$

In the optimization problem above, equation (16) states that the total disease prevalence grows at a rate determined by the net infectivity rate (i.e., the infectivity rate adjusted for each group's mobility choices and relative population shares) and the recovery rate. This is strictly related to the concept of basic reproduction number, $\mathcal{R}_{0}$, which measures the average number of secondary infections produced by a typical infectious individual introduced into a completely susceptible population [21]. Indeed, as extensively discussed in mathematical epidemiology the long-run disease dynamics crucially depends on whether the basic reproduction number is larger or smaller than one, and in our setting, such a parameter turns out to be given by the following expression:

$$
\mathcal{R}_{0}=\frac{\alpha\left(1-u_{t}^{i}\right) n^{i}+\alpha\left(1-u_{t}^{j}\right) n^{j}}{\delta} .
$$

\footnotetext{
${ }^{1}$ In the absence of a linear-quadratic structure and of $C^{1}$-regularity of the function satisfying the HamiltonJacobi-Bellman equation (HJB), obtaining closed-form solutions would not be possible. If this is the case, we might rely either on a numerical approach or on the notion of viscosity solution. Different from numerical approaches, the viscosity solutions theory permits to guarantee existence and uniqueness (via comparison principle) of the solution for problems which do not allow for classical closed-form solutions [12-14].
} 
If $\mathcal{R}_{0}<1$, that is the net infectivity rate is smaller than the recovery rate, the disease will naturally die out over time, while if $\mathcal{R}_{0}>1$, that is the net infectivity rate is higher than the recovery rate, the disease will spread and will affect a larger and larger share of the susceptible population over time. In the case of an epidemic outbreak of a disease characterized by a high $\mathcal{R}_{0}$, individuals' mobility reduction choices by lowering the net infectivity rate can effectively decrease the basic reproduction number below unity allowing to achieve a disease eradication in the long run. In the following, we shall assume that in the absence of behavioral changes (i.e., $u_{t}^{i}=u_{t}^{j}=0$ ) the disease prevalence will naturally tend to increase over time (i.e., $\mathcal{R}_{0}>1$, which occurs whenever $\alpha>\delta$ ), and in such a setting, we wish to understand whether individuals' unregulated decisions to reduce their mobility (i.e., $u_{t}^{i}, u_{t}^{j}>0$ ) may eventually reverse the disease growth pattern and lead in the long run to disease eradication (i.e., bringing $\mathcal{R}_{0}$ below unity). In particular, we wish to explore whether free-riding opportunities between groups may preclude the possibility of eradication along with how heterogeneity between groups (driven by differences at epidemiological or economic levels, captured by the groups' population size, $n^{i}$ vs $n^{j}$, and adjusted productivity, $\xi^{i}$ vs $\xi^{j}$, respectively) may affect the equilibrium outcome.

In order to put our paper in perspective, it may be useful to comment on our model's assumptions and implications. (i) We consider an infinite horizon framework as in similar macroeconomic-epidemiological settings it has been shown that the disease is likely to persist in the long run reaching an endemic state [25], and thus, it makes sense also for single individuals to plan their behavioral response to the level of disease prevalence accordingly. However, since individuals are short sighted and short lived, considering such an infinite time horizon may lead our conclusions to put excessive weight on far distant events and outcomes, which in reality would have a much lower impact on single individuals' decisions. (ii) Different forms of public policy and their implications on disease prevalence and incidence are completely ruled out to focus only on individuals' change in their behavioral patterns to contrast and limit the spread of the disease. In reality, public policy clearly plays an important role in the management of epidemic episodes by driving individuals' behavioral response and imposing stringent regulations (i.e., social distancing, lockdowns, travel bans), thus abstracting from the analysis of the interaction between public policy and individuals' mitigation strategies precludes us from analyzing several aspects of the health-economic relationship highlighted by the recent COVID-19 experience at world level. (iii) The absence of disease-induced mortality and heterogeneity between groups in several epidemiological and economic parameters limits the ability of our model to capture the peculiarities of the COVID-19 epidemics which has given rise (either directly or indirectly) to significant increases in mortality rates and significantly different impacts between groups (i.e., young vs old, men vs women).

\section{Equilibrium Outcomes}

We now analyze separately the scenarios in which the two groups' individuals do not and do cooperate in their mobility choices in order to compare the noncooperative and cooperative outcomes and analyze the implications of heterogeneity between groups in key epidemiological and economic characteristics. 


\subsection{Noncooperation}

In a noncooperative framework, the group $i$ solves problem (15) - (18) by taking the behavior of the other group $j$ as given, meaning that each group cares only about its own personal cost. It is possible to show (the proofs of all the propositions and results are presented in Appendix A) that the following result holds true.

Proposition 1 Suppose that $0<\alpha-\delta<\frac{A^{i} \alpha^{2}}{\left(\xi^{i}\right)^{2}}+\frac{A^{j} \alpha^{2}}{\left(\xi^{j}\right)^{2}}$ and $\frac{A^{i} \alpha}{\left(\xi^{i}\right)^{2}}<n^{i}<1-\frac{A^{j} \alpha}{\left(\xi^{j}\right)^{2}}$. The noncooperative Cournot-Nash mobility reduction choice for groups $i$ and $j$ along with the total infectives dynamics is, respectively, given by:

$$
\begin{aligned}
& \left(u_{t}^{i}\right)^{N}=\frac{A^{i} \alpha}{n^{i}\left(\xi^{i}\right)^{2}} \in(0,1) \\
& \left(u_{t}^{j}\right)^{N}=\frac{A^{j} \alpha}{n^{j}\left(\xi^{j}\right)^{2}} \in(0,1) \\
& i_{t}^{N}=i_{0} e^{\left[\alpha-\frac{A^{i} \alpha^{2}}{\left(\xi^{i}\right)^{2}}-\frac{A^{j} \alpha^{2}}{\left(\xi^{j}\right)^{2}}-\delta\right] t},
\end{aligned}
$$

where $A^{i}>0$ is given by (51) and $A^{j}>0$ is symmetrically determined. Furthermore, the total number of infectives monotonically falls over time, as $\dot{i}_{t}^{N}<0$.

Provided that some technical conditions hold true, Proposition 1 determines in closed form the expression for the mobility reduction choices of both groups and the dynamics of the total infectives. The mobility reduction choices of both groups turn out to be constant over time and large enough to reverse the disease growth pattern and lead to a monotonic reduction in the number of infectives. This means that despite free-riding incentives noncooperation ensures that the basic reproduction number in (19) is smaller than unity. For each group, the mobility reduction rule monotonically decreases with its own-population share: Since the population share of a group determines the cost of behavioral changes for that specific group, a higher own-group population share directly lowers its incentive to implement such changes. Apart from this intuitive result, we cannot say much about the effects of other epidemiological and economic parameters on the two groups' mobility reduction rules as the expressions in (20) and (21) are particularly cumbersome, due to the fact that the parameters $A^{i}$ and $A^{j}$ are the solutions of fourth-degree equations. Therefore, in the following we will proceed by presenting some numerical examples to visualize the impact of the main parameters on such variables.

We rely on COVID-19 data based on the Italian experience during the first epidemic wave to calibrate the model and present a real-world numerical example. Specifically, Italian estimates show that $\mathcal{R}_{0}=2.79$ and the recovery time is about 3 weeks, which imply that $\delta=0.0476$ and $\alpha=0.1328$ [25,33]). The sources of heterogeneity, $n$ and $\xi$ are instead set arbitrarily and changed within a relevant parameter range to ensure that the required technical conditions are met. In particular, we set $\xi^{j}=15$, and when we vary $\xi^{i}$, we also set $n^{i}=0.4$, while when we vary $n^{i}$, we set $\xi^{i}=1.6$. We thus assume, without loss of generality, that group $i$ is characterized by a relatively lower population share and a lower adjusted productivity than group $j$.

Figure 1 shows the impact of the group $i$ 's adjusted productivity parameter, $\xi^{i}$ (top panels), and population share, $n^{i}$ (bottom panels), on the mobility reduction rule for group $i$ (left panel), for group $j$ (middle panel) and for the difference between them (right panel). We can observe that intuitively $\left(u^{i}\right)^{N}$ falls with $\xi^{i}$, while $\left(u^{j}\right)^{N}$ increases with it: A higher adjusted productivity parameter for group $i$, on the one hand, increases group $i$ 's cost of implementing 

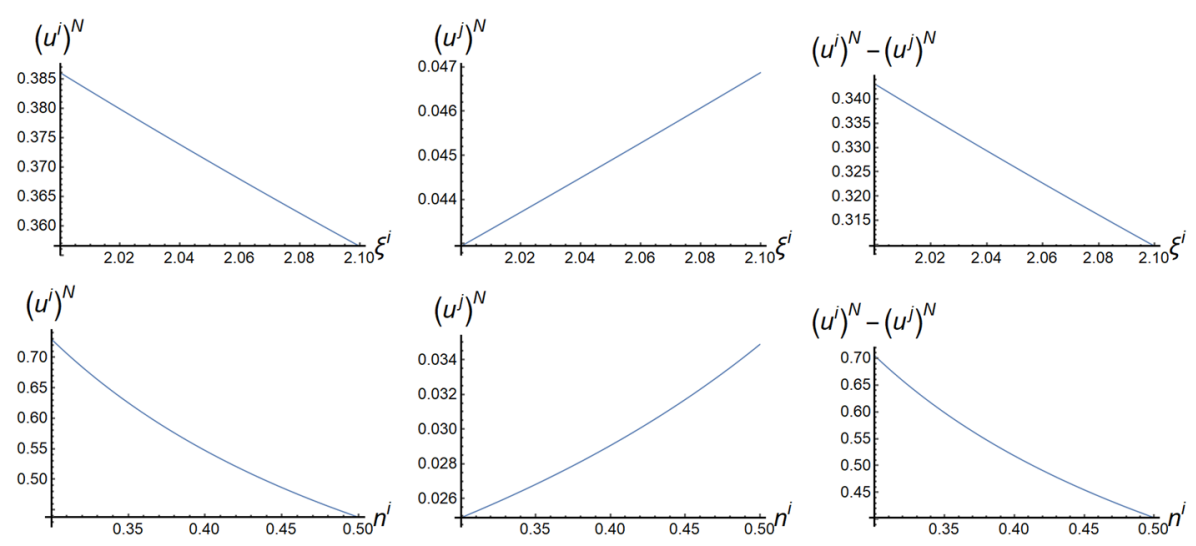

Fig. 1 Effect of the group $i$ 's adjusted productivity parameter, $\xi^{i}$ (top), and population share, $n^{i}$ (bottom), on the mobility reduction rule: $\left(u^{i}\right)^{N}$ (left), $\left(u^{j}\right)^{N}$ (center), $\left(u^{i}\right)^{N}-\left(u^{j}\right)^{N}$ (right), under noncooperation

behavioral changes lowering its incentive to reduce its mobility, and on the other hand, it increases group $j$ 's behavioral changes to compensate for the reduction in the behavioral changes of group $i$. We can also note that the mobility reduction rule is larger for group $i$, and this is due to the fact that the adjusted productivity is lower for this group which thus has relatively stronger incentive than group $j$ to implement behavioral changes, which also explains why $\left(u^{i}\right)^{N}-\left(u^{j}\right)^{N}$ falls with $\xi^{i}$. The figure shows also that, as expected, $\left(u^{i}\right)^{N}$ falls with $n^{i}$, while $\left(u^{j}\right)^{N}$ increases with it: The former result is consistent with what we have discussed earlier, while the latter follows from the fact that $n^{j}=1-n^{i}$. Exactly as we have discussed for the adjusted productivity, the mobility reduction rule is larger for group $i$, since it is characterized by a smaller population share than group $j$, and $\left(u^{i}\right)^{N}-\left(u^{j}\right)^{N}$ falls with $n^{i}$.

Therefore, we can conclude that the burden of reducing mobility patterns falls more strongly on the smaller group and the group with lower adjusted productivity (i.e., the group which allocates a lower share of its time endowment to labor activities). If we interpret the two groups as men and women, our results are consistent with the empirical evidence suggesting that women have suffered the most from the behavioral changes required to reduce the risk of infection from COVID-19. Indeed, the smaller working group in the population of most industrialized countries is represented by women, who also are those relatively less involved on the workplace due to their traditional role as primary carers for children. If we instead interpret the two groups as young and old, our results suggest that it is not possible to determine which group might suffer the most from the required behavioral changes to contain the COVID-19 epidemic, as young represent the smaller group in the working population but also that with higher involvement in labor activities.

\subsection{Cooperation}

The noncooperative framework earlier discussed is clearly not optimal as it does not minimize the joint social cost for the two groups, since the groups do not internalize the externality that their mobility choices impose on each other through the epidemic dynamics. In order to determine such a social optimum, we now focus on the cooperative setup assuming that the two groups agree to mutually determine their behavioral changes. The cooperative problem 
can be stated as follows:

$$
\begin{aligned}
\min _{u_{t}^{i}, u_{t}^{j}} & \mathcal{C}^{i}+\mathcal{C}^{j}=\int_{0}^{\infty} \frac{i_{t}^{2}\left[2+\left(\xi^{i}\right)^{2}\left(u_{t}^{i}\right)^{2}\left(n^{i}\right)^{2}+\left(\xi^{j}\right)^{2}\left(u_{t}^{j}\right)^{2}\left(n^{j}\right)^{2}\right]}{2} e^{-\rho t} d t \\
\text { s.t. } & \dot{i}_{t}=\left[\alpha\left(1-u_{t}^{i}\right) n^{i}+\alpha\left(1-u_{t}^{j}\right) n^{j}-\delta\right] i_{t} \\
& 0 \leq i_{t} \leq 1 \\
& i_{0}>0 \text { given }
\end{aligned}
$$

Similarly to the noncooperative case, also in a cooperative setting it is possible to prove the following result.

Proposition 2 Suppose that $\rho<\alpha-\delta$ and $\frac{\left(\xi^{j}\right)^{2}}{\left.2 \alpha\left[\xi^{j}\right)^{2}+\left(\xi^{i}\right)^{2}\right]}(c+\Psi)<n^{i}<1-$ $\frac{\left(\xi^{i}\right)^{2}}{2 \alpha\left[\left(\xi^{j}\right)^{2}+\left(\xi^{i}\right)^{2}\right]}(c+\Psi)$. The cooperative mobility reduction choice for groups $i$ and $j$ along with the total infectives dynamics is, respectively, given by:

$$
\begin{aligned}
& \left(u_{t}^{i}\right)^{C}=\frac{\left(\xi^{j}\right)^{2}}{2 n^{i} \alpha\left[\left(\xi^{j}\right)^{2}+\left(\xi^{i}\right)^{2}\right]}(c+\Psi) \in(0,1) \\
& \left(u_{t}^{j}\right)^{C}=\frac{\left(\xi^{i}\right)^{2}}{2 n^{j} \alpha\left[\left(\xi^{j}\right)^{2}+\left(\xi^{i}\right)^{2}\right]}(c+\Psi) \in(0,1) \\
& i_{t}^{C}=i_{0} e^{\frac{1}{2}(\rho-\Psi) t}
\end{aligned}
$$

where $c=2 \alpha-2 \delta-\rho>0$ and $\Psi=\sqrt{c^{2}+\frac{8 \alpha^{2}\left(\left(\xi^{j}\right)^{2}+\left(\xi^{i}\right)^{2}\right)}{\left(\xi^{j}\right)^{2}\left(\xi^{i}\right)^{2}}}>-c$. Furthermore, the total number of infectives monotonically falls over time, as $\dot{i}_{t}^{C}<0$.

Provided that some technical conditions are met, Proposition 2 determines in closed form the expression for the mobility reduction choices of both groups and the dynamics of the total infectives. As in the noncooperative case, individuals' mobility change is constant over time and large enough (ensuring that the basic reproduction number is smaller than unity) to achieve disease eradication in the long run, but different from the previous case we can now analytically determine the impact of the main parameters on such variables. In particular, we can show that each group's mobility reduction choice monotonically increases with the infectivity rate and the other group's adjusted productivity parameter, and it monotonically decreases with the recovery rate, its own adjusted productivity parameter and its own population share. All these results are intuitive. A higher infectivity (recovery) rate increases (decreases) the speed of disease spread and thus provides single groups with stronger (weaker) incentives to modify their behavior to reduce their exposure to infection. A higher own adjusted productivity parameter increases the group's cost of implementing behavioral changes, and thus, it directly lowers its incentive to reduce its mobility. A higher other group's adjusted productivity parameter instead increases the group's behavioral changes to compensate for the reduction in the behavioral changes of the other group. A higher population share by increasing the group's cost of implementing behavioral changes lowers its incentive to reduce its mobility.

By relying on the same parameter values earlier employed, Fig. 2 confirms these results by showing that the mobility reduction rule for group $i$ decreases with group $i$ 's adjusted productivity parameter and population share, while group $j$ 's increases with them. As in the noncooperative case, the mobility reduction rule for group $i$ is larger than group $j$, and $\left(u^{i}\right)^{C}-\left(u^{j}\right)^{C}$ falls with both $\xi^{i}$ and $n^{i}$. Exactly the same comments earlier discussed under noncooperation apply. 

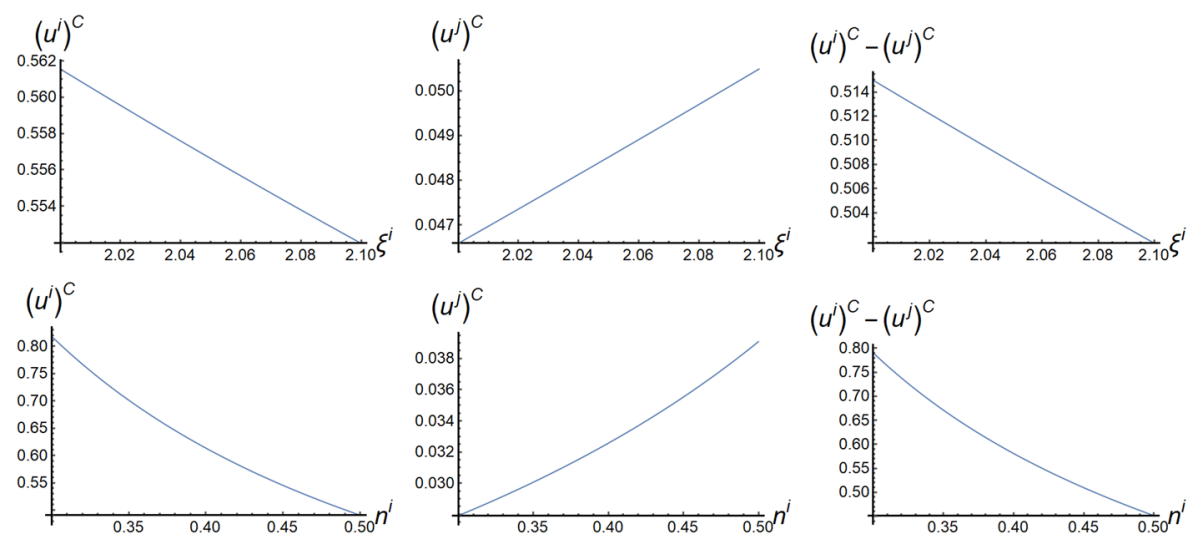

Fig. 2 Effect of the group $i$ 's adjusted productivity parameter, $\xi^{i}$ (top), and population share, $n^{i}$ (bottom), on the mobility reduction rule: $\left(u^{i}\right)^{C}$ (left), $\left(u^{j}\right)^{C}$ (center), $\left(u^{i}\right)^{C}-\left(u^{j}\right)^{C}$ (right), under cooperation
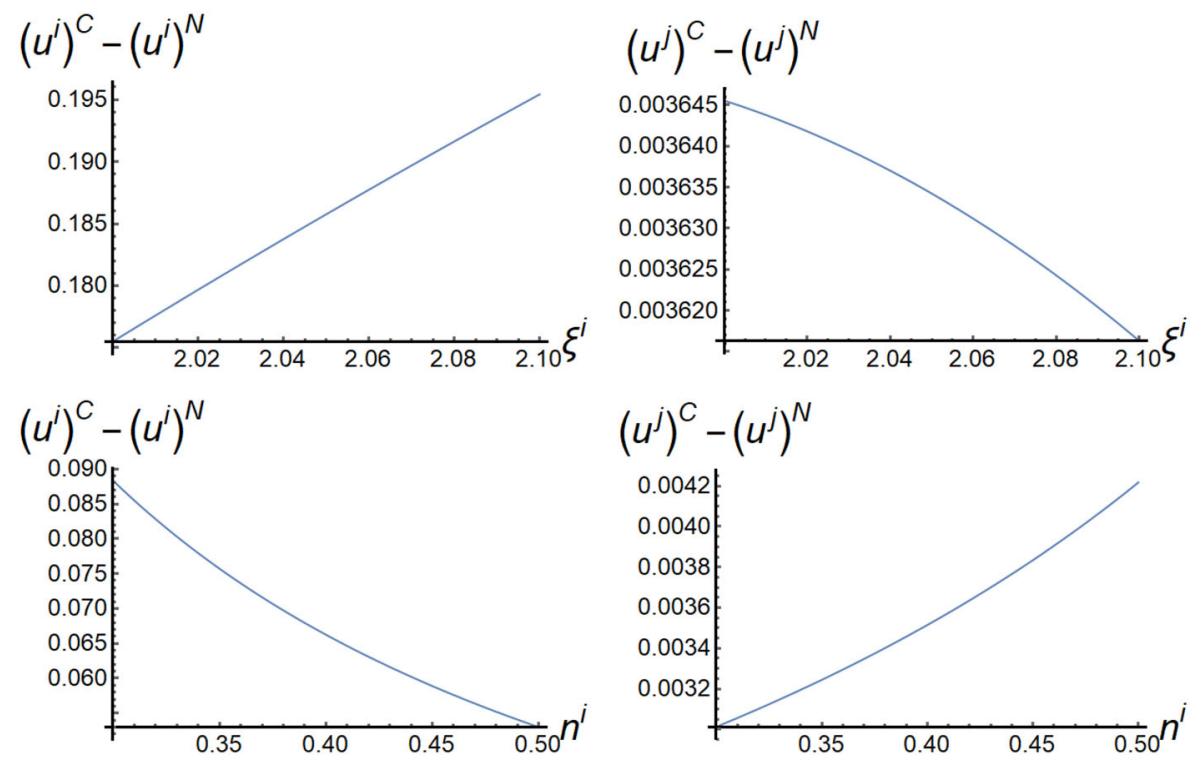

Fig. 3 Effect of the group $i$ 's adjusted productivity parameter, $\xi^{i}$ (top), and population share, $n^{i}$ (bottom), on the distortion induced by noncooperation, $\left(u_{t}^{i}\right)^{C}-\left(u_{t}^{i}\right)^{N}$ (left), and $\left(u_{t}^{j}\right)^{C}-\left(u_{t}^{j}\right)^{N}$ (right)

\subsection{Noncooperation versus Cooperation}

The difference between the cooperative and noncooperative mobility reduction choices, $\left(u_{t}^{i}\right)^{C}-\left(u_{t}^{i}\right)^{N}$ and $\left(u_{t}^{j}\right)^{C}-\left(u_{t}^{j}\right)^{N}$, determines the size of the distortion imposed by free-riding (i.e., the absence of cooperation) on epidemic dynamics. Given the cumbersomeness of the noncooperative mobility rules, it is not possible to analytically determine the determinants of such a distortion, but we will need to proceed via numerical analysis to investigate the role of the main model's parameters. 
Figure 3 shows how the size of the distortion for group $i$ (left panels) and for group $j$ (right panels) depends on the group $i$ 's adjusted productivity (top panels) and population share (bottom panels). As expected, we can observe that the distortion is positive for both groups as the failure to internalize the epidemic externalities induces each group to modify their behavioral patterns by a lower amount than what would be optimal. Moreover, the distortions for the two groups respond in a different way to a change in the parameters $\xi^{i}$ and $n^{i}:\left(u_{t}^{i}\right)^{C}-\left(u_{t}^{i}\right)^{N}$ increases with $\xi^{i}$ and falls with $n^{i}$, while $\left(u_{t}^{j}\right)^{C}-\left(u_{t}^{j}\right)^{N}$ decreases with $\xi^{i}$ and rises with $n^{i}$. These results are consistent with what we have discussed in the noncooperative and cooperative equilibrium outcomes: Under noncooperation, an increase in $\xi^{i}$ pushes group $i$ to reduce its behavioral change and dump the burden to group $j$; under cooperation such free-riding effects are internalized by redistributing this burden between groups, such to demand an increase in the efforts by group $i$ (and a decrease in those by group $j$ ) as $\xi^{i}$ increases; this is entirely reflected in the size of the distortion. A similar argument explains the response of the distortions for the two groups to a change in $n^{i}$.

The fact that the distortion induced by free-riding is positive allows us also to comment on the effectiveness of the noncooperative and cooperative strategies to contain the disease spread. Indeed, even if long-run (i.e., asymptotically) eradication is possible both under noncooperation and cooperation, since the mobility reduction rule is higher under cooperation, it follows that the number of infectives will decrease more rapidly (in finite time) when the groups coordinate their efforts. Therefore, in order to achieve effective disease containment it is essential to design appropriate policies to favor cooperation between different population groups.

\section{Symmetric Equilibrium}

In a symmetric equilibrium, the two groups are exactly identical and thus all sources of heterogeneity are ruled out, that is $\xi^{i}=\xi^{j}=\xi$ and $n^{i}=n^{j}=0.5$. In this setting, it is possible to derive a more intuitive analytical expression in the noncooperative equilibrium which allows us to perform an explicit analysis of the differences and determinants of both the noncooperative and cooperative equilibria. The noncooperative and cooperative equilibria under symmetry are characterized in the next proposition.

Proposition 3 Suppose that $\alpha-2 \delta<\rho<\alpha-\delta$ and $\xi^{2}>\frac{4 \alpha}{2 \delta-\alpha+\rho}$, and define $c=$ $2 \alpha-2 \delta-\rho>0$. Then, in a symmetric equilibrium:

- the noncooperative Cournot-Nash mobility reduction choice for both groups $i$ and $j$ along with the total infectives dynamics is, respectively, given by:

$$
\begin{aligned}
& u_{t}^{N}=\left(u_{t}^{i}\right)^{N}=\left(u_{t}^{j}\right)^{N}=\frac{1}{3 \alpha}(c+\eta) \in(0,1) \\
& i_{t}^{N}=i_{0} e^{\frac{1}{6}(c+3 \rho-2 \eta) t}>0,
\end{aligned}
$$

where $\eta=\sqrt{c^{2}+\frac{12 \alpha^{2}}{\xi^{2}}}$, and the total number of infectives monotonically falls over time, $\dot{i}_{t}^{N}<0$;

- the cooperative mobility reduction choice for both groups $i$ and $j$ along with the total infectives dynamics is instead, respectively, given by:

$$
u_{t}^{C}=\left(u_{t}^{i}\right)^{C}=\left(u_{t}^{j}\right)^{C}=\frac{1}{2 \alpha}(c+\tilde{\eta}) \in(0,1)
$$




$$
i_{t}^{C}=i_{0} e^{\frac{1}{2}(\rho-\tilde{\eta}) t}>0
$$

where $\tilde{\eta}=\sqrt{c^{2}+\frac{16 \alpha^{2}}{\xi^{2}}}$, and the total number of infectives monotonically falls over time, $i_{t}^{C}<0$.

Provided that some technical conditions hold true, Proposition 3 shows that both under noncooperation and cooperation the mobility reduction choices are large enough to reverse the disease growth pattern and lead the total number of infectives to monotonically decrease over time in order to achieve in the long-run complete disease eradication. We can also note that the noncooperative and the cooperative rules depend in the same way on the model's parameters. Specifically, it is straightforward to show that they both monotonically increase with the infectivity rate $\alpha$ and monotonically decrease with the recovery rate $\delta$ and the adjusted productivity parameter $\xi$. All these results are intuitive and consistent with what earlier discussed in the nonsymmetric case. A higher infectivity (recovery) rate increases (decreases) the speed of disease spread and thus provides single individuals with stronger (weaker) incentives to modify their behavior to reduce their exposure to infection. A higher $\xi$ increases the cost of implementing behavioral changes, and thus, it directly lowers their incentive to reduce their mobility.

Proposition 3 allows us also to directly compare the noncooperative and the cooperative rules for the mobility reduction choices and for the evolution of the total infectives share. Indeed, straightforward algebra suggests that the size of the distortion imposed by noncooperation is given by the following expression:

$$
u_{t}^{C}-u_{t}^{N}=\frac{c}{6 \alpha}+\sqrt{\frac{c^{2}}{4 \alpha^{2}}+\frac{4}{\xi^{2}}}-\sqrt{\frac{c^{2}}{9 \alpha^{2}}+\frac{4}{3 \xi^{2}}}>0,
$$

which is strictly positive as expected. As discussed in the nonsymmetric case, even if longrun (i.e., asymptotically) eradication is possible both under noncooperation and cooperation, since the mobility reduction rule is higher under cooperation, it follows that the number of infectives will decrease more rapidly (in finite time) when the groups coordinate their efforts. Indeed, as confirmed by the following expression, the difference between the cooperative and noncooperative number of infectives is strictly negative:

$$
i_{t}^{C}-i_{t}^{N}=i_{0}\left[e^{\frac{1}{2}(\rho-\widetilde{\eta}) t}-e^{\frac{1}{6}(c+3 \rho-2 \eta) t}\right]<0,
$$

suggesting that the effects due to the distortion induced by noncooperation are reflected also in the epidemic dynamics. Moreover, it is possible to show that the distortion in (34) depends on the main parameters exactly in the same way in which the noncooperative and the cooperative mobility reductions rules do; that is, it monotonically increases with the infectivity rate and monotonically decreases with both the recovery rate and the adjusted productivity parameter. This suggests that these parameters have a relatively stronger effect on the noncooperative rules than on the cooperative ones, and thus, their difference perfectly mimics their behavior. Therefore, factors increasing the pace of the disease spread (the infectivity rate $\alpha$ ) widen the wedge between the cooperative and noncooperative solutions, while factors decreasing its pace (the recovery rate $\delta$ and the adjusted productivity $\xi$ ) reduce it.

From Proposition 3, we can also determine the evolution of the infectives in the two groups. Indeed, by replacing the expression for the total number of infectives in (11), since the mobility reduction rules $u_{t}^{N}$ and $u_{t}^{C}$ are constant over time, we obtain: 


$$
\begin{aligned}
& \left(i_{t}^{i}\right)^{N}=\frac{\alpha n\left(1-u_{t}^{N}\right)\left(i_{0}^{i}+i_{0}^{j}\right)}{\frac{1}{6}(c+3 \rho-2 \eta)+\delta}\left[e^{\left(\frac{1}{6}(c+3 \rho-2 \eta)+\delta\right) t}-1\right] e^{-\delta t}+i_{0}^{i} e^{-\delta t} \\
& \left(i_{t}^{i}\right)^{C}=\frac{\alpha n\left(1-u_{t}^{C}\right)\left(i_{0}^{i}+i_{0}^{j}\right)}{\frac{1}{2}(\rho-\widetilde{\eta})+\delta}\left[e^{\left(\frac{1}{2}(\rho-\widetilde{\eta})+\delta\right) t}-1\right] e^{-\delta t}+i_{0}^{i} e^{-\delta t},
\end{aligned}
$$

in the noncooperative and cooperative cases, respectively. By substituting the total number of infectives in (12) and repeating the calculations, we obtain the same expression with swapped superscripts $i$ and $j$. With some algebra, it is possible to show that, as expected, the difference between the cooperative and noncooperative infective shares is strictly negative:

$$
\left(i_{t}^{i}\right)^{C}-\left(i_{t}^{i}\right)^{N}=n\left(i_{0}^{i}+i_{0}^{j}\right)\left[e^{\left(\frac{1}{2}(2 \delta+\rho-\widetilde{\eta})-\delta\right) t}-e^{\left(\frac{1}{3}(\alpha+2 \delta+\rho-\eta)-\delta\right) t}\right]<0 ;
$$

that is, the effects due to the distortion from free-riding affect not only the dynamics of the total infectives but also the evolution of the number of infectives in each single group.

\section{Conclusion}

The ongoing COVID-19 epidemic is ravaging the entire world showing more clearly than ever that health and macroeconomic conditions are mutually related. Apart from its dramatic consequences on economic production capabilities and the increase in mortality, the most striking effect of the epidemic is related to its highly heterogeneous impacts on different population groups (young and old, men and women). In order to shed some light on the possible determinants and implications of such heterogeneous effects, we develop a two-group differential game in which the interactions between groups determine the overall prevalence of an infectious disease, which in turn affects the level of economic activity. Individuals may lower their disease exposure by reducing their mobility, but since changing mobility patterns is costly, each group has an incentive to free ride negatively affecting the chances of disease containment at the aggregate level. We determine and compare the groups' mobility choices, along with their implications on disease prevalence, under noncooperation and cooperation, quantifying the inefficiency induced by the failure of single groups to internalize the externality that their mobility choices impose on each other through the epidemic dynamic. We show that in all scenarios the burden of reducing mobility patterns falls more strongly on the group characterized by a smaller population share and lower adjusted productivity (i.e., lower share of time allocated to labor activities), but cooperation leads to a redistribution of the burden between groups. We also show that long-run eradication may be possible even in the absence of coordination, but coordination leads to a faster reduction in the number of infectives in finite time, and that the wedge between the cooperative and noncooperative solutions increases with the factors increasing the pace of the disease spread (i.e., the infectivity rate) and decreases with those reducing it (the recovery rate and the adjusted productivity parameter).

To the best of our knowledge, this is the first paper analyzing the implications of strategic interactions between groups in a macroeconomic-epidemiological setup. For the sake of analytical tractability, we have developed a stylized early epidemic framework abstracting from several key elements (i.e., disease-induced mortality, heterogeneity in the disease transmission and recovery between groups, public health policy measures) of the COVID-19 epidemic experience. Extending the analysis to account for such factors, which would enrich 
our framework and increase its ability to provide real world policy recommendations, is left for future research.

\section{A Technical Appendix}

\section{A.1 Noncooperation}

By defining $V^{i}$ the value function associated with the problem (15) - (18), the pair $\left(i_{t}, u_{t}^{i}\right)$ is its solution if $V^{i}$ solves the following HJB equation:

$$
\rho V^{i}\left(i_{t}\right)+\max _{u_{t}^{i}}\left\{-\frac{i_{t}^{2}}{2}\left[1+\left(\xi^{i}\right)^{2}\left(u_{t}^{i}\right)^{2}\left(n^{i}\right)^{2}\right]-D V^{i}\left(i_{t}\right) \dot{i}_{t}\right\}=0,
$$

where $D V^{i}\left(i_{t}\right)$ is the first derivative of the value function $V^{i}$ with respect to $i_{t}$. The Hamiltonian $H\left(i_{t}, u_{t}^{i}, D V^{i}\left(i_{t}\right)\right)$ associated with the optimal control problem above is:

$$
H\left(i_{t}, u_{t}^{i}, D V^{i}\left(i_{t}\right)\right)=-\frac{i_{t}^{2}}{2}\left[1+\left(\xi^{i}\right)^{2}\left(u_{t}^{i}\right)^{2}\left(n^{i}\right)^{2}\right]-D V^{i}\left(i_{t}\right) \dot{i}_{t},
$$

and assuming $V^{i}\left(i_{t}\right)=\frac{A^{i} i_{t}^{2}}{2}, A^{i} \in \mathbb{R}$, we can apply the first-order condition $\partial H / \partial u_{t}^{i}=0$. Hence, assuming $i_{t} \neq 0$, we get:

$$
u_{t}^{i}=\frac{A^{i} \alpha}{n^{i}\left(\xi^{i}\right)^{2}} .
$$

Similarly, the control of the group $j$ obtained with similar reasoning as before yields:

$$
u_{t}^{j}=\frac{A^{j} \alpha}{n^{j}\left(\xi^{j}\right)^{2}} .
$$

By plugging the expressions of $V^{i}\left(i_{t}\right),(39)$ and (40) into (37), we get

$$
\frac{\alpha^{2}\left(A^{i}\right)^{2}}{\left(\xi^{i}\right)^{2}}+A^{i}\left(-2 \alpha+\rho+2 \delta+\frac{2 A^{j} \alpha^{2}}{\left(\xi^{j}\right)^{2}}\right)-1=0 .
$$

Now, denoting by $c=2 \alpha-2 \delta-\rho$, the solutions of (41) are the following

$$
\begin{aligned}
& A^{i}=\frac{\left(\xi^{i}\right)^{2}}{2 \alpha^{2}}\left(c-\frac{2 A^{j} \alpha^{2}}{\left(\xi^{j}\right)^{2}}+\sqrt{\left(-c+\frac{2 A^{j} \alpha^{2}}{\left(\xi^{j}\right)^{2}}\right)^{2}+\frac{4 \alpha^{2}}{\left(\xi^{i}\right)^{2}}}\right), \\
& A^{i}=\frac{\left(\xi^{i}\right)^{2}}{2 \alpha^{2}}\left(c-\frac{2 A^{j} \alpha^{2}}{\left(\xi^{j}\right)^{2}}-\sqrt{\left(-c+\frac{2 A^{j} \alpha^{2}}{\left(\xi^{j}\right)^{2}}\right)^{2}+\frac{4 \alpha^{2}}{\left(\xi^{i}\right)^{2}}}\right) .
\end{aligned}
$$

By substituting (39) and (40) into (16) and by integrating with respect to $t$, we get

$$
i_{t}=i_{0} e^{\left(\alpha-\frac{A^{i} \alpha^{2}}{\left(\xi^{i}\right)^{2}}-\frac{A^{j} \alpha^{2}}{\left(\xi^{j}\right)^{2}}-\delta\right) t} .
$$

The transversality condition reads as follows:

$$
\lim _{t \rightarrow+\infty} e^{-\rho t} V^{i}\left(i_{t}\right)=\lim _{t \rightarrow+\infty} e^{\left(2 \alpha-\frac{2 A^{i} \alpha^{2}}{\left(\xi^{i}\right)^{2}}-\frac{2 A^{j} \alpha^{2}}{\left(\xi^{j}\right)^{2}}-2 \delta-\rho\right) t} A^{i} \frac{i_{0}^{2}}{2}=0,
$$


which is verified if and only if:

$$
2 \alpha-\frac{2 A^{i} \alpha^{2}}{\left(\xi^{i}\right)^{2}}-\frac{2 A^{j} \alpha^{2}}{\left(\xi^{j}\right)^{2}}-2 \delta-\rho<0 .
$$

It can be proved that (46) is satisfied with $A^{i}$ as in (42).

By repeating the same analysis for group $j$ following the same approach presented above for group $i$, we assume that $V^{j}\left(i_{t}\right)=\frac{A^{j} i_{t}^{2}}{2}, A^{j} \in \mathbb{R}$, where $A^{j}$ is given by:

$$
A^{j}=\frac{\left(\xi^{j}\right)^{2}}{2 \alpha^{2}}\left(c-\frac{2 A^{i} \alpha^{2}}{\left(\xi^{i}\right)^{2}}+\sqrt{\left(-c+\frac{2 A^{i} \alpha^{2}}{\left(\xi^{i}\right)^{2}}\right)^{2}+\frac{4 \alpha^{2}}{\left(\xi^{j}\right)^{2}}}\right)
$$

At this point, we need to determine the value of the coefficients $A^{i}$ and $A^{j}$ in order to obtain a unique expression of the optimal dynamics $i_{t}$ and of the controls $u_{t}^{i}$ and $u_{t}^{j}$. The system between (42) and (47) reads as follows:

$$
\left\{\begin{array}{l}
A^{i}=\frac{\left(\xi^{i}\right)^{2}}{2 \alpha^{2}}\left(c-\frac{2 A^{j} \alpha^{2}}{\left(\xi^{j}\right)^{2}}+\sqrt{\left(-c+\frac{2 A^{j} \alpha^{2}}{\left(\xi^{j}\right)^{2}}\right)^{2}+\frac{4 \alpha^{2}}{\left(\xi^{i}\right)^{2}}}\right) \\
A^{j}=\frac{\left(\xi^{j}\right)^{2}}{2 \alpha^{2}}\left(c-\frac{2 A^{i} \alpha^{2}}{\left(\xi^{i}\right)^{2}}+\sqrt{\left(-c+\frac{2 A^{i} \alpha^{2}}{\left(\xi^{i}\right)^{2}}\right)^{2}+\frac{4 \alpha^{2}}{\left(\xi^{j}\right)^{2}}}\right)
\end{array}\right.
$$

By plugging the expression of $A^{j}$ in the first equation of (48), we get the fourth-degree equation:

$$
\frac{3 \alpha^{4}\left(A^{i}\right)^{4}}{\left(\xi^{i}\right)^{4}}-\frac{4 \alpha^{2} c\left(A^{i}\right)^{3}}{\left(\xi^{i}\right)^{2}}+\left(A^{i}\right)^{2}\left(c^{2}+\frac{4 \alpha^{2}}{\left(\xi^{j}\right)^{2}}-\frac{2 \alpha^{2}}{\left(\xi^{i}\right)^{2}}\right)-1=0 .
$$

whose solutions are given by the cumbersome expression below:

$$
A^{i}=\frac{1}{6}\left\{\frac{2 c\left(\xi^{i}\right)^{2}}{\alpha^{2}} \pm \sqrt{\mathcal{S}^{i}} \pm 3 \sqrt{\mathcal{L}_{1}^{i}+\mathcal{L}_{2}^{i}}\right\}
$$

where

$$
\begin{aligned}
& \mathcal{L}_{1}^{i}=\frac{4 c^{3}\left(\xi^{i}\right)^{6}}{9 \alpha^{6} S^{i}}+\frac{8\left(\xi^{i}\right)^{2}\left(\left(\xi^{j}\right)^{2}-2\left(\xi^{i}\right)^{2}\right)}{9 \alpha^{2}\left(\xi^{j}\right)^{2}} \\
& \mathcal{L}_{2}^{i}=\frac{4 c\left(\xi^{i}\right)^{4}\left(\left(\xi^{j}\right)^{2}\left(c S^{i}+6\right)-12\left(\xi^{i}\right)^{2}\right)-S\left(f^{i}+\left(p^{i}\right)^{2 / 3}\right)\left(p^{i}\right)^{-\frac{1}{3}}}{9 \alpha^{4}\left(\xi^{j}\right)^{2} S^{i}}, \\
& \mathcal{S}^{i}=\frac{2\left(\xi^{i}\right)^{2}\left(2 \alpha^{2}\left(\left(\xi^{j}\right)^{2}-2\left(\xi^{i}\right)^{2}\right)+c^{2}\left(\xi^{j}\right)^{2}\left(\xi^{i}\right)^{2}\right)+f^{i}\left(p^{i}\right)^{-\frac{1}{3}}+\left(p^{i}\right)^{\frac{1}{3}}}{\alpha^{4}\left(\xi^{j}\right)^{2}}, \\
& f^{i}=\left(\xi^{i}\right)^{4}\left(\left(c^{2}\left(\xi^{j}\right)^{2}\left(\xi^{i}\right)^{2}-2 \alpha^{2}\left(\left(\xi^{j}\right)^{2}-2\left(\xi^{i}\right)^{2}\right)\right)^{2}-36 \alpha^{4}\left(\xi^{j}\right)^{4}\right)
\end{aligned}
$$

along with

$$
\begin{aligned}
& p^{i}=6 \sqrt{3} \sqrt{g^{i}}+\left(\xi^{i}\right)^{6}(-224 \alpha^{6}\left(\xi^{j}\right)^{6}-6\left(\xi^{i}\right)^{4}\left(4 \alpha^{3}\left(\xi^{j}\right)+\alpha c^{2}\left(\xi^{j}\right)^{3}\right)^{2} \\
&\left.+\left(\xi^{i}\right)^{6}\left(4 \alpha^{2}+c^{2}\left(\xi^{j}\right)^{2}\right)^{3}+96 \alpha^{4}\left(\xi^{j}\right)^{4}\left(\xi^{i}\right)^{2}\left(5 \alpha^{2}-c^{2}\left(\xi^{j}\right)^{2}\right)\right),
\end{aligned}
$$


where

$$
\begin{aligned}
g^{i}=\alpha^{4}\left(\xi^{j}\right)^{4}\left(\xi^{i}\right)^{12}( & 256 \alpha^{6} c^{2}\left(\xi^{j}\right)^{2}\left(\xi^{i}\right)^{2}\left(2\left(\xi^{j}\right)^{6}-3\left(\xi^{j}\right)^{4}\left(\xi^{i}\right)^{2}-3\left(\xi^{j}\right)^{2}\left(\xi^{i}\right)^{4}+2\left(\xi^{i}\right)^{6}\right) \\
& -c^{8}\left(\xi^{j}\right)^{8}\left(\xi^{i}\right)^{8}+96 \alpha^{4}\left(\left(\xi^{j}\right)^{4}-\left(\xi^{j}\right)^{2}\left(\xi^{i}\right)^{2}+\left(\xi^{i}\right)^{4}\right) \\
& \left.\times\left(8 \alpha^{4}\left(\left(\xi^{j}\right)^{4}-\left(\xi^{j}\right)^{2}\left(\xi^{i}\right)^{2}+\left(\xi^{i}\right)^{4}\right)+c^{4}\left(\xi^{j}\right)^{4}\left(\xi^{i}\right)^{4}\right)\right) .
\end{aligned}
$$

Among the four solutions (51), let us consider the one with all positive factors for being simultaneously respected the transversality condition of $A^{i}$ and $A^{j}$, that is:

$$
A^{i}=\frac{1}{6}\left\{\frac{2 c\left(\xi^{i}\right)^{2}}{\alpha^{2}}+\sqrt{\mathcal{S}^{i}}+3 \sqrt{\mathcal{L}_{1}^{i}+\mathcal{L}_{2}^{i}}\right\}
$$

Then, we get the unique expression of $A^{j}$ which coincide with the chosen $A^{i}$ unless to exchange the role of $\xi^{i}$ and $\xi^{j}$. In conclusion, consider the chosen $A^{i}$ (51) and the corresponding $A^{j}$, we obtain the closed-form expressions of the controls and the state variable, given by (20), (21) and (22), respectively.

\section{Cooperation}

By defining $V$ the value function associated with the problem (23) - (26), the pair $\left(i_{t}, u_{t}^{i}\right)$ is its solution if $V$ solves the following HJB equation:

$$
\rho V\left(i_{t}\right)+\max _{u_{t}^{i}, u_{t}^{j}}\left\{-\frac{i_{t}^{2}}{2}\left[2+\left(\xi^{i}\right)^{2}\left(u_{t}^{i}\right)^{2}\left(n^{i}\right)^{2}+\left(\xi^{j}\right)^{2}\left(u_{t}^{j}\right)^{2}\left(n^{j}\right)^{2}\right]-D V\left(i_{t}\right) \dot{i}_{t}\right\}=0 .
$$

The Hamiltonian $H\left(i_{t}, u_{t}^{i}, u_{t}^{j}, D V\left(i_{t}\right)\right)$ associated with the optimal control problem above is given by:

$$
H\left(i_{t}, u_{t}^{i}, u_{t}^{j}, D V\left(i_{t}\right)\right)=-\frac{i_{t}^{2}}{2}\left[2+\left(\xi^{i}\right)^{2}\left(u_{t}^{i}\right)^{2}\left(n^{i}\right)^{2}+\left(\xi^{j}\right)^{2}\left(u_{t}^{j}\right)^{2}\left(n^{j}\right)^{2}\right]-D V\left(i_{t}\right) \dot{i}_{t} .
$$

Assuming that $V\left(i_{t}\right)=\frac{A i_{t}^{2}}{2}, A \in \mathbb{R}$, we can apply the first-order conditions to get the expression of the controls, i.e., $\nabla H=0$, which is equivalent to impose that $\left(\frac{\partial H}{\partial u_{t}^{i}}, \frac{\partial H}{\partial u_{t}^{j}}\right)=$ $(0,0)$. Then, we have

$$
\left\{\begin{array}{l}
i_{t}^{2}\left(\xi^{i}\right)^{2}\left(n^{i}\right)^{2} u_{t}^{i}-A i_{t}\left(\alpha n^{i} i_{t}\right)=0 \\
i_{t}^{2}\left(\xi^{j}\right)^{2}\left(n^{j}\right)^{2} u_{t}^{j}-A i_{t}\left(\alpha n^{j} i_{t}\right)=0
\end{array}\right.
$$

from which follows that

$$
\left\{\begin{array}{l}
u_{t}^{i}=\frac{A \alpha}{n^{i}\left(\xi^{i}\right)^{2}}, \\
u_{t}^{j}=\frac{A \alpha}{n^{j}\left(\xi^{j}\right)^{2}} .
\end{array}\right.
$$


Note that in order to derive $u_{t}^{i}$ and $u_{t}^{j}$ we suppose that $i_{t} \neq 0$. By plugging (54) in (24) and by integrating with respect to $t$, we get

$$
i_{t}=i_{0} e\left(\alpha-A \alpha^{2} \frac{\left(\xi^{i}\right)^{2}+\left(\xi^{j}\right)^{2}}{\left(\xi^{i}\right)^{2}\left(\xi^{j}\right)^{2}}-\delta\right) t .
$$

By plugging the expressions of $V\left(i_{t}\right), u_{t}^{i}$ and $u_{t}^{j}$ into the HJB (52), we obtain

$$
\alpha^{2}\left(\frac{1}{\left(\xi^{j}\right)^{2}}+\frac{1}{\left(\xi^{i}\right)^{2}}\right) A^{2}+(-2 \alpha+\rho+2 \delta) A-2=0 .
$$

Now, denoting by

$$
c=2 \alpha-2 \delta-\rho, \quad \Psi=\sqrt{c^{2}+\frac{8 \alpha^{2}\left(\left(\xi^{j}\right)^{2}+\left(\xi^{i}\right)^{2}\right)}{\left(\xi^{j}\right)^{2}\left(\xi^{i}\right)^{2}},}
$$

the solutions of (56) are the following

$$
\begin{aligned}
& A=\frac{\left(\xi^{i}\right)^{2}\left(\xi^{j}\right)^{2}}{2 \alpha^{2}\left(\left(\xi^{i}\right)^{2}+\left(\xi^{j}\right)^{2}\right)}(c+\Psi), \\
& A=\frac{\left(\xi^{i}\right)^{2}\left(\xi^{j}\right)^{2}}{2 \alpha^{2}\left(\left(\xi^{i}\right)^{2}+\left(\xi^{j}\right)^{2}\right)}(c-\Psi) .
\end{aligned}
$$

From the transversality condition, we get which $A$ as to be considered:

$$
\lim _{t \rightarrow+\infty} e^{-\rho t} V\left(i_{t}\right)=\lim _{t \rightarrow+\infty} e^{\left(2 \alpha-2 A \alpha^{2} \frac{\left(\xi^{i}\right)^{2}+\left(\xi^{j}\right)^{2}}{\left(\xi^{i}\right)^{2}\left(\xi^{j}\right)^{2}}-2 \delta-\rho\right) t} A \frac{i_{0}^{2}}{2}=0
$$

if and only if

$$
2 \alpha-2 A \alpha^{2} \frac{\left(\xi^{i}\right)^{2}+\left(\xi^{j}\right)^{2}}{\left(\xi^{i}\right)^{2}\left(\xi^{j}\right)^{2}}-2 \delta-\rho<0
$$

It is easy to verify that (59) is satisfied taking $A$ as in (57). In conclusion, by replacing (57) into (55) and (54) we get the closed-form expressions of the controls and the state variable, given by (27), (28) and (29), respectively. The derivatives of the cooperative rule for the mobility reduction choices for group $i$ (those for group $j$ are symmetrically determined) with respect to the main parameters are given by the following expressions:

$$
\begin{aligned}
\frac{\partial\left(u_{t}^{i}\right)^{C}}{\partial \alpha}= & \frac{\left(\xi^{j}\right)^{2}(2 \delta+\rho)(c+\Psi)}{2 \alpha^{2} n^{i} \Psi\left[\left(\xi^{j}\right)^{2}+\left(\xi^{i}\right)^{2}\right]}>0, \\
\frac{\partial\left(u_{t}^{i}\right)^{C}}{\partial \delta}= & -\frac{\left(\xi^{j}\right)^{2}(c+\Psi)}{\left.\alpha n^{i} \Psi\left[\xi^{j}\right)^{2}+\left(\xi^{i}\right)^{2}\right]}<0, \\
\frac{\partial\left(u_{t}^{i}\right)^{C}}{\partial \xi^{i}}= & -\frac{\left(\xi^{j}\right)^{2}\left(\left(\xi^{i}\right)^{4} \Psi(c+\Psi)+4 \alpha^{2}\left[\left(\xi^{j}\right)^{2}+\left(\xi^{i}\right)^{2}\right]\right)}{\alpha n^{i} \Psi\left(\xi^{i}\right)^{3}\left[\left(\xi^{j}\right)^{2}+\left(\xi^{i}\right)^{2}\right]^{2}}<0, \\
\frac{\partial\left(u_{t}^{i}\right)^{C}}{\partial \xi^{j}}= & \frac{\left(\xi^{i}\right)^{2}\left(\xi^{j}\right)^{2}\left(c \sqrt{(2 \delta+\rho)(2 \delta+\rho-4 \alpha)+\alpha^{2}\left(4+8\left(\frac{\left.\left.\left(\xi^{j}\right)^{2}+\left(\xi^{i}\right)^{2}\right)\right)}{\left(\xi^{j}\right)^{2}\left(\xi^{i}\right)^{2}}\right)\right.}+(2 \delta+\rho)(2 \delta+\rho-4 \alpha)\right)}{\alpha n^{i} \Psi\left(\xi^{j}\right)\left[\left(\xi^{j}\right)^{2}+\left(\xi^{i}\right)^{2}\right]^{2}} \\
& +\frac{4 \alpha^{2}\left(\left(\xi^{j}\right)^{2}+\left(\xi^{i}\right)^{2}\left(1+\left(\xi^{j}\right)^{2}\right)\right)}{\alpha n^{i} \Psi\left(\xi^{j}\right)\left[\left(\xi^{j}\right)^{2}+\left(\xi^{i}\right)^{2}\right]^{2}}>0, \\
\frac{\partial\left(u_{t}^{i}\right)^{C}}{\partial n^{i}}= & -\frac{\left(\xi^{j}\right)^{2}(c+\Psi)}{2 \alpha\left(n^{i}\right)^{2}\left[\left(\xi^{j}\right)^{2}+\left(\xi^{i}\right)^{2}\right]}<0 .
\end{aligned}
$$




\section{A.3 Symmetry}

We now assume that $V^{i}=V^{j}$, which implies that $A^{i}=A^{j}=A$. In this setting, the controls (39) and (40) take the same form given by:

$$
u_{t}^{i}=u_{t}^{j}=\frac{2 A \alpha}{\xi^{2}}
$$

respectively. By plugging the expressions of $V\left(i_{t}\right)=\frac{A i_{t}^{2}}{2}$ and (60) into (37), we get:

$$
\frac{3 \alpha^{2}}{\xi^{2}} A^{2}+(-2 \alpha+\rho+2 \delta) A-1=0
$$

Denoting by:

$$
c=2 \alpha-2 \delta-\rho, \quad \eta=\sqrt{c^{2}+\frac{12 \alpha^{2}}{\xi^{2}}}
$$

the solutions of (61) are:

$$
\begin{aligned}
& A=\frac{\xi^{2}}{6 \alpha^{2}}(c+\eta), \\
& A=\frac{\xi^{2}}{6 \alpha^{2}}(c-\eta) .
\end{aligned}
$$

The transversality condition is verified if and only if:

$$
2 \alpha-\frac{4 \alpha^{2}}{\xi^{2}} A-2 \delta-\rho<0 .
$$

It is easy to verify that (64) is satisfied taking $A$ as in (62). In conclusion, we obtain the closedform expressions of the control and the state variable, given by (30) and (31), respectively. The derivatives of the noncooperative rule for the mobility reduction choices with respect to the main parameters are given by the following expressions:

$$
\begin{aligned}
\frac{\partial u_{t}^{N}}{\partial \xi} & =-\frac{4 \alpha}{\xi^{2} \sqrt{c^{2} \xi^{2}+12 \alpha^{2}}}<0 \\
\frac{\partial u_{t}^{N}}{\partial \alpha} & =\frac{(2 \delta+\rho)\left(c \xi+\sqrt{c^{2} \xi^{2}+12 \alpha^{2}}\right)}{3 \alpha^{2} \sqrt{c^{2} \xi^{2}+12 \alpha^{2}}}>0 \\
\frac{\partial u_{t}^{N}}{\partial \delta} & =-\frac{2\left(c \xi+\sqrt{c^{2} \xi^{2}+12 \alpha^{2}}\right)}{3 \alpha \sqrt{c^{2} \xi^{2}+12 \alpha^{2}}}<0
\end{aligned}
$$

In the cooperative case, the first-order conditions of the Hamiltonian associated with (52) yield $\nabla H=0$ if and only if:

$$
\left\{\begin{array}{l}
u_{t}^{i}=\frac{2 A \alpha}{\xi^{2}}, \\
u_{t}^{j}=\frac{2 A \alpha}{\xi^{2}} .
\end{array}\right.
$$

By substituting the expression of $V\left(i_{t}\right)$ and (65) into (52), we have:

$$
\frac{2 \alpha^{2}}{\xi^{2}} A^{2}+(-2 \alpha+\rho+2 \delta) A-2=0 .
$$


Denoting by:

$$
\tilde{\eta}=\sqrt{c^{2}+\frac{16 \alpha^{2}}{\xi^{2}}},
$$

and taking $c$ as before, the solutions of (66) are

$$
\begin{aligned}
& A=\frac{\xi^{2}}{4 \alpha^{2}}(c+\tilde{\eta}), \\
& A=\frac{\xi^{2}}{4 \alpha^{2}}(c-\tilde{\eta}) .
\end{aligned}
$$

As in the noncooperative case, it can be verified that the transversality condition requires to consider $A$ as in (67). Finally, the closed-form expressions of the control and the state variable are given by (32) and (33), respectively. The derivatives of the cooperative rule for the mobility reduction choices with respect to the main parameters are given by the following expressions:

$$
\begin{aligned}
\frac{\partial u_{t}^{C}}{\partial \xi} & =-\frac{8 \alpha}{\xi^{2} \sqrt{c^{2} \xi^{2}+16 \alpha^{2}}}<0, \\
\frac{\partial u_{t}^{C}}{\partial \alpha} & =\frac{(2 \delta+\rho)\left(c \xi+\sqrt{c^{2} \xi^{2}+16 \alpha^{2}}\right)}{2 \alpha^{2} \sqrt{c^{2} \xi^{2}+16 \alpha^{2}}}>0, \\
\frac{\partial u_{t}^{C}}{\partial \delta} & =-\frac{c \xi+\sqrt{c^{2} \xi^{2}+16 \alpha^{2}}}{\alpha \sqrt{c^{2} \xi^{2}+16 \alpha^{2}}}<0 .
\end{aligned}
$$

The derivatives of the distortion imposed by noncooperation (34) with respect to the main parameters are given by the following expressions:

$$
\begin{aligned}
& \frac{\partial\left(u_{t}^{C}-u_{t}^{N}\right)}{\partial \xi}=\frac{4 \alpha}{\xi^{2}}\left(\frac{1}{\sqrt{c^{2} \xi^{2}+12 \alpha^{2}}}-\frac{2}{\sqrt{c^{2} \xi^{2}+16 \alpha^{2}}}\right)<0, \\
& \frac{\partial\left(u_{t}^{C}-u_{t}^{N}\right)}{\partial \alpha}=\frac{2 \delta+\rho}{6 \alpha^{2}}\left(1-\frac{2 \xi(2 \alpha-2 \delta-\rho)}{\sqrt{(2 \alpha-2 \delta-\rho)^{2} \xi^{2}+12 \alpha^{2}}}+\frac{3 \xi(2 \alpha-2 \delta-\rho)}{\sqrt{(2 \alpha-2 \delta-\rho)^{2} \xi^{2}+16 \alpha^{2}}}\right)>0, \\
& \frac{\partial\left(u_{t}^{C}-u_{t}^{N}\right)}{\partial \delta}=-\frac{1}{3 \alpha}\left(1-\frac{2 \xi(2 \alpha-2 \delta-\rho)}{\sqrt{(2 \alpha-2 \delta-\rho)^{2} \xi^{2}+12 \alpha^{2}}}+\frac{3 \xi(2 \alpha-2 \delta-\rho)}{\sqrt{(2 \alpha-2 \delta-\rho)^{2} \xi^{2}+16 \alpha^{2}}}\right)<0 .
\end{aligned}
$$

\section{References}

1. Acemoglu D, Chernozhukov V, Werning I, Whinston MD (2020) A multi-risk SIR model with optimally targeted lockdown, Am Econ Rev: Insights (Forthcoming)

2. Alon T, Doepke M, Olmstead-Rumsey J, Tertilt M (2020) This time it's different: the role of women's employment in a pandemic recession. CEPR Discussion Paper 15149

3. Alvarez FE, Argente D, Lippi F (2020) A simple planning problem for COVID-19 lockdown. Am Econ Rev: Insights (Forthcoming)

4. Angelucci M, Angrisani M, Bennett DM, Kapteyn A, Schaner SG (2020) Remote work and the heterogeneous impact of COVID-19 on employment and health. NBER Working Paper 27749

5. Atkeson A (2020) What will be the economic impact of COVID-19 in the US? Rough estimates of disease scenarios. NBER Working Paper 26867

6. Belot M, Choi S, Tripodi E, van den Broek-Altenburg E, Jamison JC, Papageorge NW (2020) Unequal consequences of COVID-19 across age and income: representative evidence from six countries. IZA Discussion Paper 13366 
7. Caselli FG, Grigoli F, Sandri D, Spilimbergo A (2020) Mobility under the COVID-19 pandemic: asymmetric effects across gender and Age. IMF Working Paper 2020/282

8. Chakraborty S, Papageorgiou C, Pérez Sebastián F (2010) Diseases, infection dynamics and development. J Monet Econ 57:859-872

9. Chetty R, Friedman JN, Hendren N, Stepner M (2020) How did COVID-19 and stabilization policies affect spending and employment? A new real-time economic tracker based on private sector data. NBER Working Paper 27431

10. Chowell G, Sattenspiel L, Bansal S, Viboud C (2016) Mathematical models to characterize early epidemic growth: a review. Phys Life Rev 18:66-97

11. Codagnone C, Bogliacino F, Gomez C, Charris R, Montealegre F, Liva G, Lupianez-Villanueva F, Folkvord F, Veltri GA (2020) Assessing concerns for the economic consequence of the COVID-19 response and mental health problems associated with economic vulnerability and negative economic shock in Italy, Spain, and the United Kingdom. PLoS ONE 15:e0240876

12. Crandall MG, Lions P-L (1983) Viscosity solutions of Hamilton-Jacobi equations. Trans Am Math Soc 277:1-42

13. Crandall MG, Evans LC, Lions P-L (1984) Some properties of viscosity solutions of Hamilton-Jacobi equations. Trans Am Math Soc 282:487-502

14. Crandall MG, Ishii H, Lions P-L (1987) Uniqueness of viscosity solutions of Hamilton-Jacobi equations revisited. J Math Soc Jpn 39:581-596

15. Crossley TF, Fisher P, Low H (2021) The heterogeneous and regressive consequences of COVID-19: evidence from high quality panel data. J Public Econ 193:104334

16. Eichenbaum M, Rebelo S, Trabandt M (2020) The macroeconomics of epidemics. NBER Working Paper 26882

17. Gersovitz M, Hammer JS (2004) The economical control of infectious diseases. Econ J 114:1-27

18. Goenka A, Liu L, Nguyen MH (2014) Infectious diseases and economic growth. J Math Econ 50:34-53

19. Glaeser EL, Gorback CS, Redding SJ (2020) How much does COVID-19 increase with mobility? Evidence from New York and four other US cities. NBER, Working Paper 27519

20. Glover A, Heathcote J, Krueger D, Rios-Rull J-V (2020) Health versus wealth: on the distributional effects of controlling a pandemic. CEPR Discussion Paper 14606

21. Hethcote HW (2000) The mathematics of infectious diseases. SIAM Rev 42:599-653

22. Hupkau C, Petrangolo B (2020) Work, care and gender during the COVID-19 crisis. CEPR Discussion Paper 15358

23. Jorgensen S, Zaccour G (2019) Optimal pricing and advertising policies for a one-time entertainment event. J Econ Dyn Control 100:395-416

24. Kermack WO, McKendrick AG (1927) A contribution to the mathematical theory of epidemics. Proc R Soc Lond Ser A 115:700-721

25. La Torre D, Liuzzi D, Marsiglio S (2021) Epidemics and macroeconomic outcomes: social distancing intensity and duration. J Math Econ 93:102473

26. La Torre D, Malik T, Marsiglio S (2020) Optimal control of prevention and treatment in a basic macroeconomic-epidemiological model. Math Soc Sci 108:100-108

27. Liu S, Yang L, Zhang C, Xiang Y-T, Liu Z, Hu S, Zhang B (2020) Online mental health services in China during the COVID-19 outbreak. Lancet 7:e17-e18

28. Lopez AD, Mathers CD, Ezzati M, Jamison DT, Murray CJL (2006) Global burden of disease and risk factors. New York, Oxford University Press

29. Ma J (2020) Estimating epidemic exponential growth rate and basic reproduction number. Infect Disease Model 5:129-141

30. Marsiglio S, Masoudi N (2021) Transboundary pollution control and competitiveness concerns in a twocountry differential game. Environ Model Assess (Forthcoming)

31. Philipson T (2000) Economic epidemiology and infectious disease. In: Cuyler AJ, Newhouse JP (eds) Handbook of health economics, vol 1B. Amsterdam, North Holland, pp 1761-1799

32. Reluga TC (2010) Game theory of social distancing in response to an epidemic. PLoS Comput Biol 6:e1000793

33. Remuzzi A, Remuzzi G (2020) COVID-19 and Italy: what next? Lancet 395:1225-1228

34. World Health Organization (2009) World Health Statistics 2009. Available http://www.who.int/gho/ publications/world_health_statistics/EN_WHS09_Full.pdf

35. World Health Organization (2020) "Immunity passports" in the context of COVID-19-Scientific brief. Available https://www.who.int/news-room/commentaries/detail/immunity-passports-in-the-context-ofcovid-19 
Publisher's Note Springer Nature remains neutral with regard to jurisdictional claims in published maps and institutional affiliations.

\section{Authors and Affiliations}

\section{Davide La Torre $^{1}$ (D) Danilo Liuzzi ${ }^{2} \cdot$ Rosario Maggistro $^{3} \cdot$ Simone Marsiglio $^{4}$}

$凶$ Davide La Torre

davide.latorre@skema.edu

Danilo Liuzzi

danilo.liuzzi@unimi.it

Rosario Maggistro

rosario.maggistro@deams.units.it

Simone Marsiglio

simone.marsiglio@unipi.it

1 SKEMA Business School and Université Côte d'Azur, Sophia Antipolis, France

2 Department of Economics, Management and Quantitative Methods, University of Milan, Milan, Italy

3 Department of Economics, Business, Mathematics and Statistics "B. de Finetti”, University of Trieste, Trieste, Italy

4 Department of Economics and Management, University of Pisa, Pisa, Italy 